\title{
Nanostructuring discotic molecules on ITO support
}

\author{
H C Hesse ${ }^{1}$, D Lembke ${ }^{1}$, L Dössel ${ }^{2}$, X Feng ${ }^{2}$, K Müllen ${ }^{2}$ \\ and $\mathrm{L}$ Schmidt-Mende ${ }^{1}$ \\ ${ }^{1}$ Department of Physics and Center for NanoScience (CeNS), \\ Ludwig-Maximilians-University Munich, Amalienstraße 54, 80799 Munich, Germany \\ ${ }^{2}$ Max Planck Institute for Polymer Research, Ackermannweg 10, 55128 Mainz, Germany \\ E-mail: hesse@physik.lmu.de
}

\begin{abstract}
Patterning of organic compounds on a nanometer length scale is of great interest for solar applications: defined control over the donor-acceptor interface will allow design of an optimized nano-morphology promoting exciton separation and reducing charge recombination. Herein we present an imprinting technique using anodized alumina oxide (AAO) hard templates as stamps. We show an exact pattern transfer of the AAO structures into a solution processable hexa-peri-hexabenzocoronene (HBC), a discotic small molecule with acrylate moieties which is polymerized in situ. Film fabrication is realized for a variety of nanowire dimensions on square centimeter areas. The fabrication directly on conductive glass support and control over the formation of a dense barrier layer render this approach appealing for the fabrication of fully organic nanostructured photovoltaic devices.
\end{abstract}

\section{Introduction}

Photovoltaics based on organic materials are currently investigated intensively. The potentially low production cost and versatile processability are major advantages of organic photovoltaics (OPV) when compared with their inorganic counterparts. Polymer-fullerene solar cells have achieved remarkable efficiency of over $7 \%$ due to the introduction of the bulk heterojunction principle, optimization of film forming procedures and careful choice of materials $[1,2]$. The large surface area of donor-acceptor blend mixtures allows harvesting of excitons generated throughout the active layer and after annealing treatments sufficient percolation pathways are formed for free charges to exit the device [3]. Recent investigations have shown that for further performance increase precise control of nano-morphology and molecular alignment are necessary providing improved charge carrier mobility and reduced recombination loss in the OPV device [4-6]. Small molecular weight materials with high purity and self-assembly processes may help to reduce defects in the bulk phase achieving the above mentioned goals [4].
Hexabenzocoronenes are appealing donor materials for organic solar cells with self-organizing properties [7, 8]. Aligned stacks of these discotic molecules can exhibit remarkably high charge carrier mobility and can function as 1D organic nanowires providing excellent electronic transport [9, 10]. Precise control over ordering and nanoarchitecture is necessary to take advantage of these properties in an OPV device.

An approach to gain increased influence on the morphology is the formation of concentration gradients or 'interdigitated' interfaces of donor and acceptor, e.g. by subsequent deposition and controlled inter-diffusion of the compounds [11]. Reduced recombination and high charge carrier mobility have been shown for such devices [11-13]. Precise control of the interfacial architecture on the nanometer length scale will allow minimization of recombination losses while maintaining the electrical properties of the pristine materials.

Different approaches have shown to hold promise for the controlled nano-patterning of organic materials. Bottom up approaches are often based on the self-assembly of block 

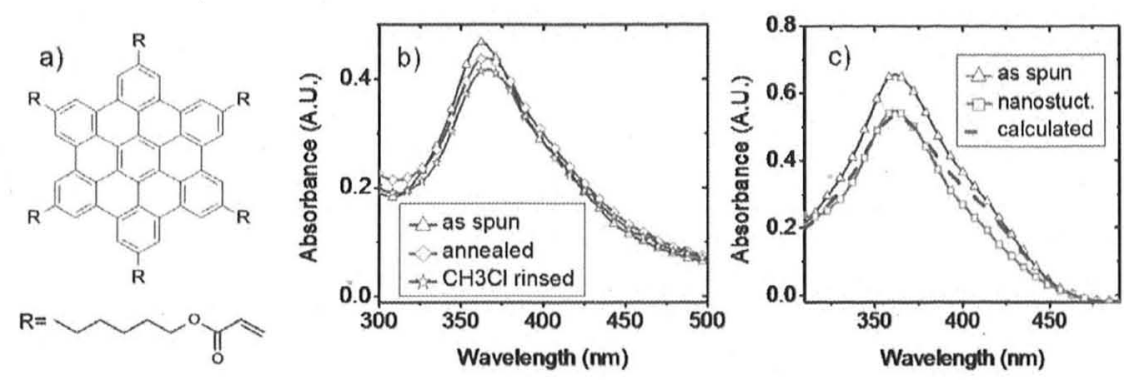

Figure 1. HBC absorption spectra. (a) HBC-cross-linkable chemical formula. (b) HBC-cross-linkable on quartz glass as spun, rinsed with $\mathrm{CH}_{3} \mathrm{Cl}$ and after annealing. (c) $\mathrm{HBC}$-cross-linkable on ITO support as spun and after nanostructuring. The dashed line shows the calculated absorption (pore diameter $75 \mathrm{~nm}$, inter-pore distance $100 \mathrm{~nm}$, imprint down to the substrate).

copolymers or polymer brushes [14, 15]. More independent control of nanostructure dimensions and molecular packing can be gained by top down methods, e.g. hard template production and a transfer of this structure into the organic material [16]. Nanostructured templates can be subsequently filled with organic material by various techniques, e.g. spin coating or electrophoretic deposition $[17,18]$.

In this work an imprinting technique is used to directly transfer the template structures of an anodized alumina oxide membrane into the organic material. Polymerization of the organic compound is induced at elevated temperatures and used to stabilize the compound irreversibly. This allows precise structuring of the donor material directly on the conductive glass support. The template can be fully removed after the structure transfer and subsequent solution deposition of an organic acceptor without dissolution of the donor material becomes feasible.

\section{Materials and methods}

The synthesis and properties of the cross-linkable discotic material used are described elsewhere $[19,20]$; the chemical formula is depicted in figure 1(a). Differential scanning calorimetry and $\mathrm{x}$-ray studies revealed a crystalline order at room temperature, a phase transition to a liquid crystalline state at $112^{\circ} \mathrm{C}$ and an irreversible polymerization reaction of the acrylate moieties starting at approximately $150^{\circ} \mathrm{C}[19]$.

Thin films of the compound solubilized in chloroform were directly deposited on indium tin oxide (ITO) coated glass substrates (pgo, $<20 \Omega \mathrm{cm}^{-2}$ ) or quartz glass (ITOS, Suprasil, Grade II) using spin coating techniques. Substrates were previously cleaned in acetone and isopropanol followed by a plasma cleaning treatment (Diener Femto).

Absorption spectra were recorded using an Agilent 8453 UV-vis absorption spectrometer.

AAO templates were fabricated on high purity $\mathrm{Al}$ foil (99.999\%, Goodfellow) by two-step anodization as described elsewhere in detail [21, 22]. Pore depth, inter-pore distance and diameter can be controlled independently by proper choice of electrolyte solution, anodization voltage and time and subsequent pore widening procedures $[22,23]$. In this study both sulfuric acid and oxalic acid were used as electrolyte solutions with anodization voltages of $25 \mathrm{~V}$ and $40 \mathrm{~V}$, respectively. Pore widening was conducted in a $\mathrm{H}_{3} \mathrm{PO}_{4}$ bath (5 wt\%) for 5-60 min yielding different pore diameters. A fluorinated silane was covalently bound to the AAO templates in order to reduce adhesion with the organic material [24].

The pattern of the AAO membranes produced was subsequently transferred into the organic material by an imprinting step using a temperature and pressure controlled bench press. The cross-linkable material is polymerized in the confinement of the AAO template by slowly ramping both the substrate temperature and the applied pressure to $170^{\circ} \mathrm{C}$ and 100 bar, respectively. After maintaining the sample for $2 \mathrm{~h}$ at this elevated temperature the pressure is released and the samples are cooled back to room temperature at a rate of $1^{\circ} \mathrm{C} \min ^{-1}$.

Subsequently the aluminum support and AAO membrane are carefully removed by immersion of the sample in dilute $\mathrm{CuCl}_{2}$ solution $(0.2 \mathrm{M})$ and phosphoric acid bath (5 wt\%).

Atomic force microscopy (AFM) scans were conducted in tapping mode on several samples using a Veeco Dimension 3100 scanner and silicon scanning tips $\left(f_{0}=200-500 \mathrm{kHz}\right)$.

Scanning electron microscopy (SEM) was conducted on a LEO DSM 982. For better resolution a thin layer of Au was sputter coated on the samples prior to imaging.

\section{Results and discussion}

In a first experiment homogeneous thin films of the organic compound were deposited on quartz substrates. Annealing of the films above the polymerization temperature renders the material insoluble in organic solvents. Consequently absorption spectra recorded from a film as spun, after an annealing treatment at $170{ }^{\circ} \mathrm{C}$ and after subsequent rinsing in chloroform, show almost neglectable deviation (figure 1(b)).

Figure 1(c) shows absorption spectra of a film before and after the imprinting. The change in absorption is attributed to a reallocation of the material by imprinting: simple application of the Lambert-Beer law for flat and structured films explains an extinction dependent decrease of absorption (see supporting information available at stacks.iop.org/Nano/ 22/055303/mmedia).

The dashed line shows the calculated absorption assuming an imprint down to the substrate and a 3/4 quotient for pillar height/pillar spacing as derived from SEM scans on the sample. 

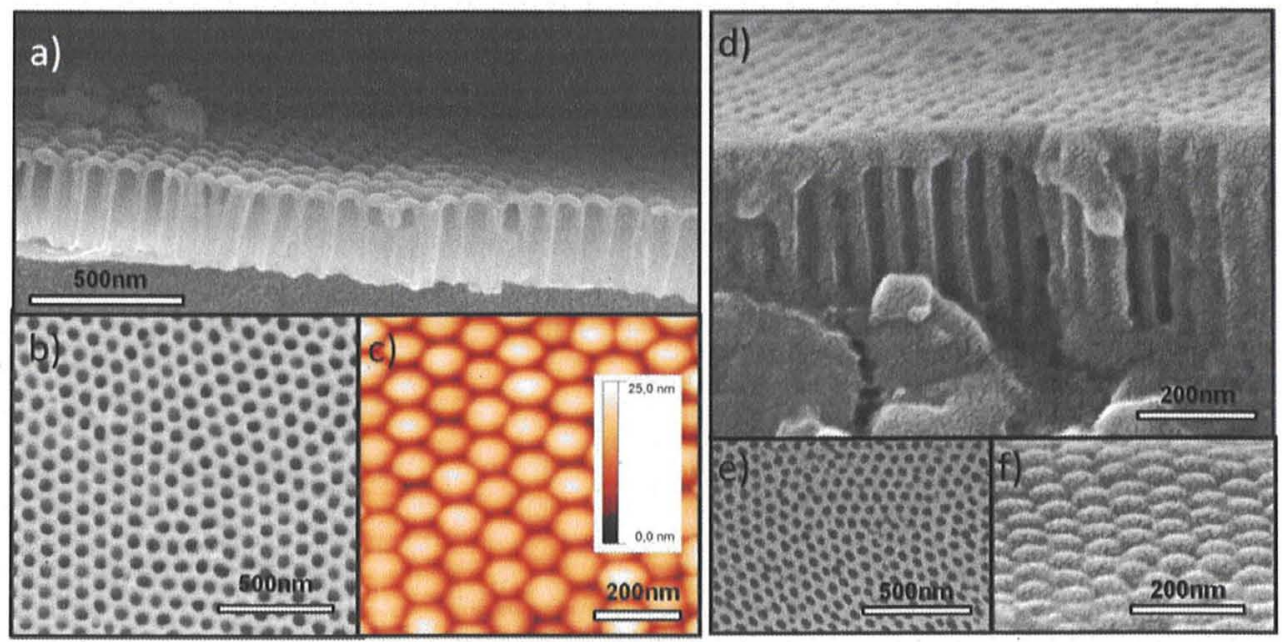

Figure 2. Exemplary set of AAO templates used for the imprinting. (a)-(c) Anodization in oxalic acid at $40 \mathrm{~V}$ for $150 \mathrm{~s}, 30 \mathrm{~min}$ pore widening. (d)-(f) Anodization in sulfuric acid at $25 \mathrm{~V}$ for $300 \mathrm{~s}, 10$ min pore widening.

The slight deviation from the measured spectrum after imprint is attributed to light scattering effects, material alignment due to the imprinting and a marginal surface roughness of the spin coated film.

The pore dimensions and quality of the AAO templates used for this study have been monitored by multiple SEM and AFM scans (figure 2). Bottom views of the AAO membranes (figures 2(b) and (e)) show the typical hexagonal ordering and reveal pore diameter and inter-pore distance. Top views (figures 2(c) and (f)) and cross-sections (figures 2(a) and (d)) were also performed on the membranes after removal of the Al support and subsequent transfer onto an ITO support. These scans reveal the exact pore depth, diameter and quality of hexagonal ordering. Clear differences are visible for samples produced at low and high anodization voltages in sulfuric acid (figures 2(d)-(f)) and oxalic acid (figures 2(a)(c)), respectively. Image analysis reveals $60 \mathrm{~nm}$ (sulfuric acid) and $100 \mathrm{~nm}$ (oxalic acid) inter-pore distances for the samples shown, in good accordance with literature [22, 23].

In order to produce the nanostructured HBC films, about $100 \mathrm{~nm}$ thick layers of the organic material are spin coated on ITO glass and immediately transferred to the press. High pressure and a stepwise increase of the temperature are applied to the sandwich of substrate and AAO on aluminum support in order to first induce area-wide template filling and subsequently polymerization of the organic material. After slow cooling back to room temperature the AAO is found to adhere to the substrate. The sandwich structures are then transferred into a self-made holder equipped with gaskets which is used to selectively immerse the aluminum top surface of the sandwich structure in the $\mathrm{CuCl}_{2}$ solution. The gasket system provides protection of the ITO layer from exposure to the acidic and basic solutions. Figure 3(a) shows a photograph of a nanostructured film after template removal-the HBC film is recovered after the imprinting process with visibly no defects. Only at the edges, where the gasket system did not prevent the exposure to the acidic and basic solutions, the ITO is dissolved and subsequently the organic film starts to delaminate.
The resulting nanostructured films have been analyzed by atomic force microscopy (AFM) and scanning electron microscopy (SEM) techniques (figure 3). A SEM top view (figure 3(b)) shows hexagonally ordered pillars of the polymerized organic material. The pattern is an exact replica of the imprinted AAO structure showing identical ordering and dimensions. An AFM scan visualizes the surface of the wires showing the identical structures (figure 3(c)) and reveals that the resulting pillars show only small deviations in height. It should be mentioned that due to tip convolution effects of the high aspect ratio structures the height trace does not cover valuable information on the height of individual pillars. Figure 3(d) shows a SEM cross-section view. The free standing wires can be seen directly attached to the ITO support. Furthermore, we also analyzed the edge of a sample (figure 3(e)). On the left-hand side the pure ITO surface is visible and to the lower right the organic nanowires are attached. At the borderline some wires have been ripped off allowing visualization of both aspect ratio and ordering within one micrograph. For the structures visualized in figure 3(e) we find nanowires of $300 \mathrm{~nm}$ length and a diameter of $90 \mathrm{~nm}$ resulting in an aspect ratio of 3.33 .

To further analyze the structure transfer from inorganic hard template into the organic material we also monitored the structures through the AAO etching processes (figure 4). Interestingly we can precisely determine the level of filling for individual membrane pores (figures 4(a) and (b)) - for the aspect ratio shown the organic material reaches up to about $4 / 5$ of the AAO template depth. Enclosed air inside the membrane pores is compressed throughout the imprinting process and due to increasing temperature polymerization of the initially soft organic material takes place rendering it more viscous and finally immobile. Simple calculations applying the ideal gas law reveal that at about 12 bar air pressure of the enclosed air the material flow into the pores is stopped for the sample shown. It should be mentioned that the pore filling is apparently also stopped if the AAO reaches down to the ITO support regardless of the pressure and temperature applied. 


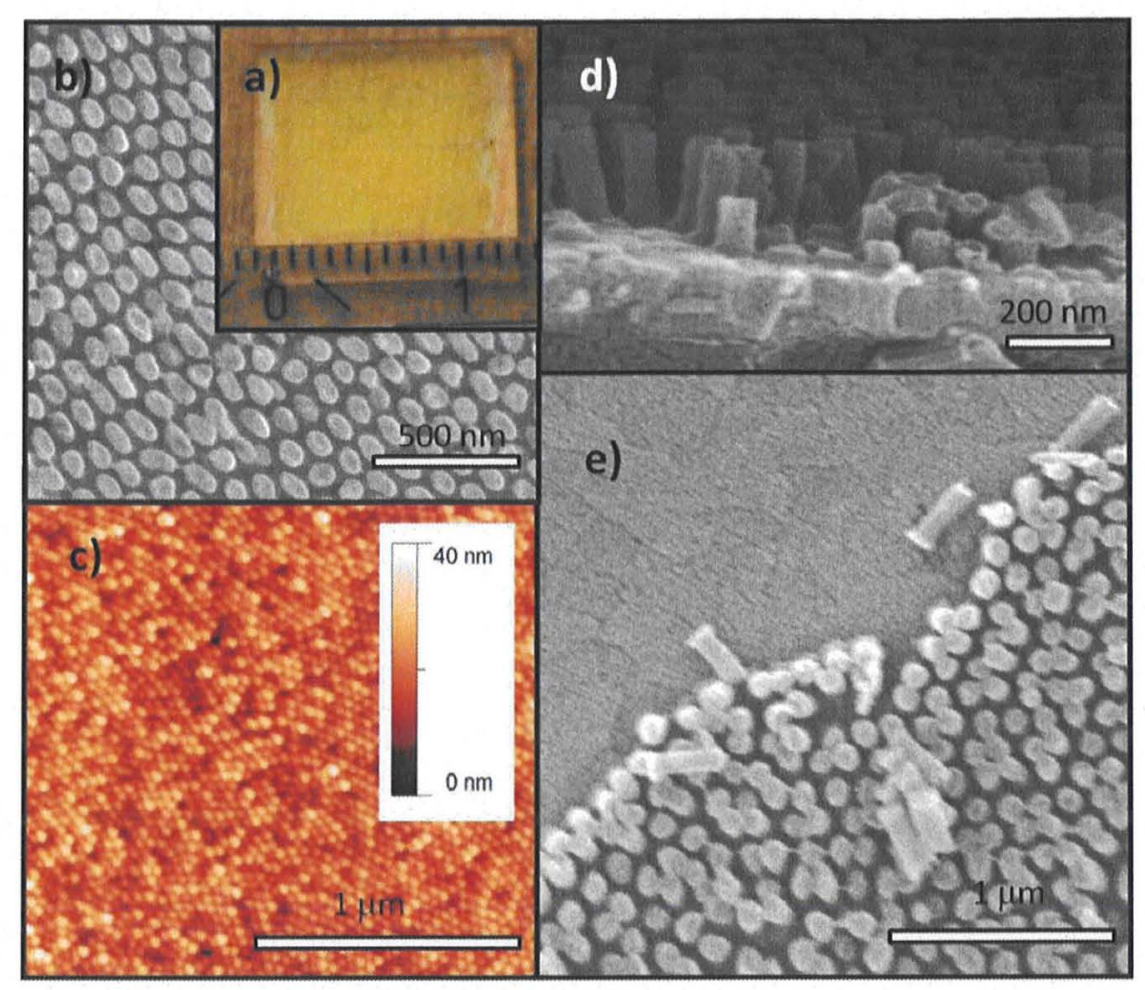

Figure 3. (a) Photograph of a nanoimprinted HBC film showing almost full coverage and defect free film production. (b)-(d) SEM and AFM images of HBC nanostructures on ITO support after the imprint and template dissolution.

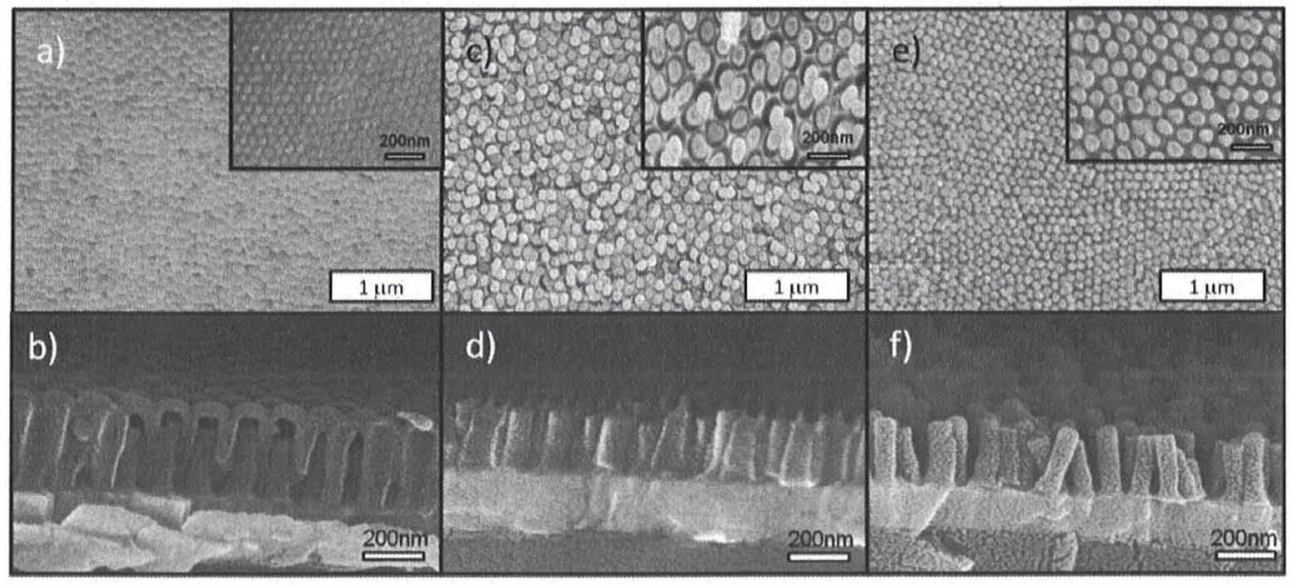

Figure 4. Analysis of the HBC filling level and AAO etching evolution by SEM images. Top views (a), (c), (e) and cross-sections (b), (d), (f) of different production steps.

An easier and more precise control over the formation of a barrier layer can be gained by using a two-step polymerization as described below. Images taken after etching only a few nanometers of $\mathrm{AAO}$ (figures 4(c) and (d)) reveal a complete removal of the barrier layer and demonstrate that almost all pores are filled with the organic material. Figures 4(e) and (f) show the same sample after a complete removal of the AAO template.

Using a selection of different AAO templates we produced organic nanowire arrays with various dimensions. Figure 5 shows a selection of the polymerized wires in SEM top and side views. We achieved fully free standing wires with an aspect ratio of 2.2 with an $\mathrm{AAO}$ anodized at $40 \mathrm{~V}$ in oxalic acid for $60 \mathrm{~s}$ and a pore widening for $30 \mathrm{~min}$ (figure 5(a)). When increasing the nanowire length the structures start to bend (figure 5(b), oxalic acid at $40 \mathrm{~V}$ for $80 \mathrm{~s}$, pore widening $20 \mathrm{~min}$; aspect ratio of 2.7). Further increase in length and reduction of diameter leads to aggregation of the wires upon drying in air (figure 5(c), sulfuric acid at $25 \mathrm{~V}$ for $150 \mathrm{~s}$, pore widening 10 min; aspect ratio of 5.5). Bundles of several wires can 


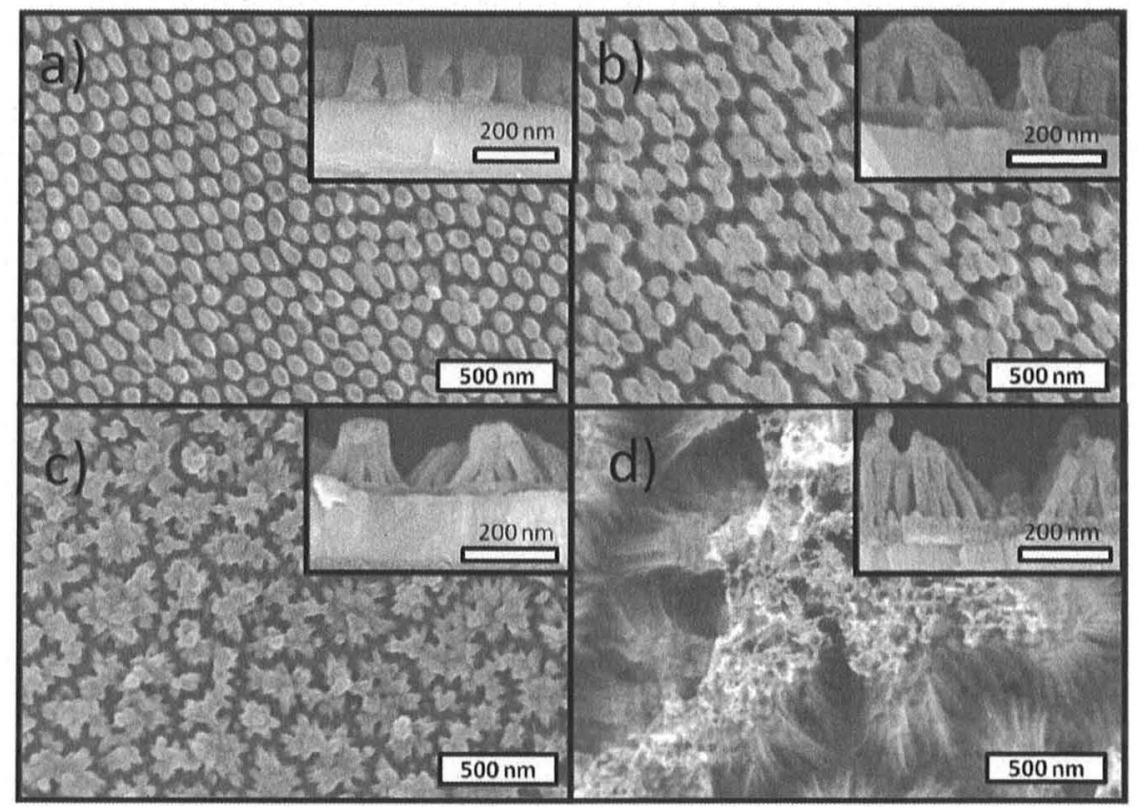

Figure 5. SEM top views and cross-sections of HBC nanowires on ITO support with differing aspect ratios.

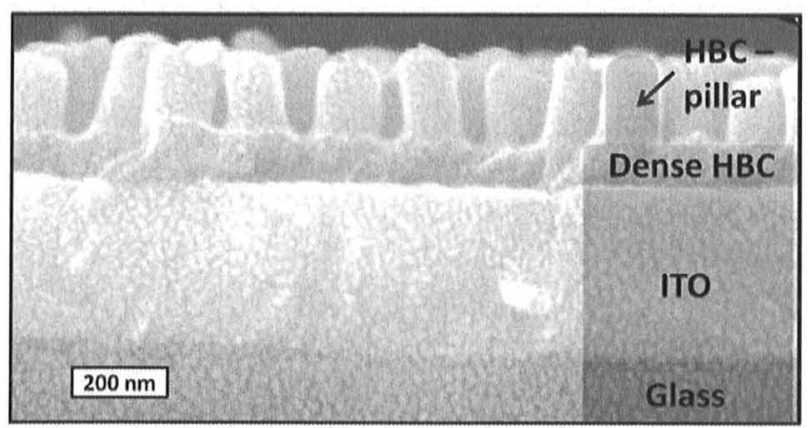

Figure 6. SEM cross-section micrograph showing $\mathrm{HBC}$ nanowires and a dense supporting HBC barrier layer fabricated by two-step polymerization as described in the text.

be found when imprinting with very small pores (figure 5(d), sulfuric acid at $25 \mathrm{~V}$ for $300 \mathrm{~s}, 5 \mathrm{~min}$ pore widening). The resulting nanowires have a length of $180 \mathrm{~nm}$ and a diameter of only $22 \mathrm{~nm}$. This results in an aspect ratio of 8.1. Even at the highest aspect ratio of over 8 the wires are still bound tightly to the ITO surface and form big bundles rather than completely collapsing. Critical point drying might help to yield free standing structures also at higher aspect ratio [25, 26].

A little modification in the fabrication process-a twostep polymerization-also allowed us to precisely control the formation of a dense HBC layer underneath the patterned film (figure 6): in a first step a thin layer of the organic material is deposited by spin coating on the ITO support. This flat film is polymerized and immobilized by thermal annealing at $170^{\circ} \mathrm{C}$ resulting in a dense barrier layer. Subsequently a second layer of the organic material was deposited from solution followed by an imprinting step as described above. Only the second layer consisting of monomeric HBC molecules is strongly affected by this imprinting step. By variations of the spin coating parameters for the two deposition steps we can individually and precisely control the layer thicknesses of the non-structured dense barrier layer and the formation of the nanopillars on this supporting layer respectively. For the sample shown in figure 6 we realized an array of $180 \mathrm{~nm}$ long wires grown on a $30 \mathrm{~nm}$ thick barrier. A defect free and accurately controlled growth of a barrier layer is a prerequisite for a fabrication of efficient nanostructured solar cell devices as one can ensure that donor and acceptor compounds will be only in contact with their respective electrodes. This allows very effective suppression of exciton and charge carrier recombination at the contacts.

\section{Conclusion}

Nanostructured organic films have been produced previously with other organic donor materials yielding a comparable aspect ratio to that presented herein $[17,27]$. However, in these studies the nanowires are produced by direct (over)-filling of commercial AAO templates and a subsequent lamination step on ITO via special silanes, e.g. vinyl-Si(OMe)3 [17], or directly on the ITO support using siloxane-derivatized organic materials [27]. In contrast, the technique presented here allows for a production of fully organic nanowires directly on the ITO support with precise control of a barrier layer. Intimate contact and complete grafting to the ITO substrate with no air encapsulation are ensured. Furthermore, no siloxane is necessary for ITO grafting which might alter the electronic properties of the organic compound or introduce traps in the bulk material. The processing also does not rely on distinct melting temperatures of the two organic materials as is the case for the so called double imprinting presented recently [28]. 
Additionally our new method allows the controlled formation of a thin barrier layer.

The polymerization techniques presented herein also allow freezing of the molecules in either crystalline or liquid crystalline state depending on the polymerization procedure: if acrylate moieties are polymerized at elevated temperatures as reported here the resulting films show liquid crystalline order [19]. This allows minimization of grain boundaries while maintaining the $\pi-\pi$ stacking of neighboring discotic molecules [29]. On the other hand the polymerization of the molecule could also be induced by UV-curing at lower temperatures resulting in amorphous or crystalline films which might be advantageous for some applications [19].

Investigations on solar cells consisting of the nanostructured films filled with an electron acceptor are currently underway. First tests show that only low conductivity can be observed in the thermally polymerized HBC material. On the contrary, very high charge carrier mobility has been shown for other HBC derivatives [9]. We attribute the low conductivity to insufficient alignment and also charge trapping in residual groups: a strong decrease in conductivity has been found for other $\mathrm{HBC}$ derivatives where bulky side groups are attached to the parent molecule [30, 31]. Optimization of the AAO surface treatment, the nanowire diameter and the polymerization procedure will help to yield increased alignment [4, 32]. Despite careful processing and subsequent rinsing of the $\mathrm{HBC}$ layers a decrease in conductivity could also result from adhesion of siloxane used for the AAO surface modification or other molecules used for AAO removal. Furthermore, it has been shown that on average only four out of the six acrylate moieties of the monomer will be effectively crosslinked during the polymerization procedure [19]. Nonlinked acrylate moieties will most likely act as traps, hinder defect free face to face stacking of the $\mathrm{HBC}$ cores and thus strongly limit charge carrier transport through the organic layer. The design of a $\mathrm{C} 3$-symmetric molecule like recently shown in the literature and the use of a less space demanding linker will certainly help to improve device performance [33].

We demonstrate the fabrication of free standing organic nanowires directly on ITO support. Precise control over the desired structures is achieved by using AAO templates as stamps with individual control over nanowire length, spacing and diameter. We demonstrate that the hexagonal pattern of the AAOs can be fully transferred into the organic material. The resulting structures are directly attached to the ITO support and show an aspect ratio of up to 8.1 (22 $\mathrm{nm}$ in diameter, $180 \mathrm{~nm}$ long). Nanostructured samples have been produced on square centimeters and the process presented here is easily scalable to even larger sample areas. After polymerization the patterned organic material is insoluble in common organic solvents allowing for simple and cost effective application of an acceptor compound, e.g. perylene diimide. We also demonstrate the deposition of a dense barrier layer with tunable thickness underneath the structured films by a two-step polymerization. No additional grafting material is necessary for the attachment to the ITO support rendering this approach very instructive towards the fabrication of nanostructured OPV devices with precise morphology control.

\section{Acknowledgments}

We gratefully acknowledge the German research foundation (DFG) for funding in the program 'SPP1355: elementary processes of organic photovoltaics' as well as funding in the Cluster of Excellence 'Nanosystems Initiative Munich (NIM)'. We would like to thank W Töllner and S Heiderich in the Professor Nielsch Group (University of Hamburg, Germany) for their support on AAO production. $\mathrm{H} \mathrm{C}$ Hesse thanks Christian Hundschell for support in calculating absorption spectra. We thank Professor $\mathbf{J}$ Kotthaus and Professor J Feldmann for giving access to their equipment.

\section{References}

[1] Scharber M, Mühlbacher D, Koppe M, Denk P, Waldauf C, Heeger A and Brabec C 2006 Design rules for donors in bulk-heterojunction solar cells-towards $10 \%$ energy-conversion efficiency Adv. Mater. 18 789-94

[2] Liang Y, Xu Z, Xia J, Tsai S-T, Wu Y, Li G, Ray C and Yu L 2010 For the bright future-bulk heterojunction polymer solar cells with power conversion efficiency of $7.4 \%$ Adv. Mater. 22 E135

[3] Hoppe H, Niggemann M, Winder C, Kraut J, Hiesgen R, Hinsch A, Meissner D and Sariciftci N 2004 Nanoscale morphology of conjugated polymer/fullerene-based bulk-heterojunction solar cells Adv. Funct. Mater. 14 1005-11

[4] Pisula W, Feng X and Müllen K 2010 Tuning the columnar organization of discotic polycyclic aromatic hydrocarbons Adv. Mater. 223634

[5] Street R A and Schoendorf M 2010 Interface state recombination in organic solar cells Phys. Rev. B 81205307

[6] Venkataraman D, Yurt S, Venkatraman B H and Gavvalapalli N 2010 Role of molecular architecture in organic photovoltaic cells J. Phys. Chem. Lett. 1 947-58

[7] Schmidt-Mende L, Fechtenkotter A, Mullen K, Moons E, Friend R H and MacKenzie J D 2001 Self-organized discotic liquid crystals for high-efficiency organic photovoltaics Science 293 1119-22

[8] Hesse H, Weickert J, Al-Hussein M, Dössel L, Feng X, Müllen K and Schmidt-Mende L 2010 Discotic materials for organic solar cells: effects of chemical structure on assembly and performance Sol. Energy Mater. Sol. Cells 94 560-7

[9] van de Craats A M, Warman J M, Fechtenkotter A, Brand J D, Harbison M A and Mullen K 1999 Record charge carrier mobility in a room-temperature discotic liquid-crystalline derivative of hexabenzocoronene Adv. Mater. 11 1469-72

[10] Pisula W, Menon A, Stepputat M, Lieberwirth I, Kolb U, Tracz A, Sirringhaus H, Pakula T and Mullen K 2005 A zone-casting technique for device fabrication of field-effect transistors based on discotic hexa-peri-hexabenzoeoronene Adv. Mater. 17684

[11] Schubert M, Yin C, Castellani M, Bange S, Tam T, Sellinger A, Hörhold H, Kietzke T and Neher D 2009 Heterojunction topology versus fill factor correlations in novel hybrid small-molecular/polymeric solar cells J. Chem. Phys. 130094703

[12] Chen L, Godovsky D, Inganäs O, Hummelen J, Janssens R, Svensson M and Andersson M 2000 Polymer photovoltaic devices from stratified multilayers of donor-acceptor blends Adv. Mater. 12 1367-70

[13] Drees M, Premaratne K, Graupner W, Heflin J, Davis R. Marciu D and Miller M 2002 Creation of a gradient polymer-fullerene interface in photovoltaic devices by thermally controlled interdiffusion Appl. Phys. Lett. 814607 
[14] Lee J I, Cho S H, Park S M, Kim J K, Kim J K, Yu J W, Kim Y C and Russell T P 2008 Highly aligned ultrahigh density arrays of conducting polymer nanorods using block copolymer templates Nano Lett. 8 2315-20

[15] Snaith H J, Whiting G L, Sun B, Greenham N C, Huck W T S and Friend R H 2005 Self-organization of nanocrystals in polymer brushes. Application in heterojunction photovoltaic diodes Nano Lett. 5 1653-7

[16] Steinhart M 2008 Supramolecular organization of polymeric materials in nanoporous hard templates Adv. Polym. Sci. 220 123-87

[17] Haberkorn N, Gutmann J S and Theato P 2009 Template-assisted fabrication of free-standing nanorod arrays of a hole-conducting cross-linked triphenylamine derivative: toward ordered bulk-heterojunction solar cells ACS Nano 3 1415-22

[18] Bai R, Ouyang M, Zhou R J, Shi M M, Wang M and Chen H Z 2008 Well-defined nanoarrays from an n-type organic perylene-diimide derivative for photoconductive devices Nanotechnology 19055604

[19] Kastler M, Pisula W, Davies R J, Gorelik T, Kolb U and Mullen K 2007 Nanostructuring with a crosslinkable discotic material Small 3 1438-44

[20] Zhi L J, Wu J S, Li J X, Kolb U and Mullen K 2005 Carbonization of disclike molecules in porous alumina membranes: toward carbon nanotubes with controlled graphene-layer orientation Angew. Chem. Int. Edn 44 2120-3

[21] Lee W, Ji R, Gosele U and Nielsch K 2006 Fast fabrication of long-range ordered porous alumina membranes by hard anodization Nat. Mater. 5 741-7

[22] Li A P, Muller F, Birner A, Nielsch K and Gosele U 1998 Hexagonal pore arrays with a 50-420 nm interpore distance formed by self-organization in anodic alumina $J$. Appl. Phys. $846023-6$

[23] Musselman K P, Mulholland G J, Robinson A P, Schmidt-Mende L and MacManus-Driscoll J L 2008 Low-temperature synthesis of large-area, free-standing nanorod arrays on ITO/glass and other conducting substrates Adv. Mater. 20 4470-5

[24] Beck M, Graczyk M, Maximov I, Sarwe E L, Ling T G I, Keil M and Montelius L 2002 Improving stamps for $10 \mathrm{~nm}$ level wafer scale nanoimprint lithography Microelectron. Eng. 61/62 441-8

[25] Zhu K, Vinzant T B, Neale N R and Frank A J 2007 Removing structural disorder from oriented $\mathrm{TiO}_{2}$ nanotube arrays: reducing the dimensionality of transport and recombination in dye-sensitized solar cells Nano Lett. 7 3739-46

[26] Botiz I, Martinson A B F and Darling S B 2010 Minimizing lateral domain collapse in etched poly(3-hexylthiophene)-block-polylactide thin films for improved optoelectronic performance Langmuir 26 8756-61

[27] Haberkorn N, Weber S A L, Berger R D and Theato P 2010 Template-based preparation of free-standing semiconducting polymeric nanorod arrays on conductive substrates ACS Appl. Mater. Interfaces 2 1573-80

[28] He X, Gao F, Tu G, Hasko D, Hüttner S, Steiner U, Greenham N, Friend R and Huck W 2010 Formation of nanopatterned polymer blends in photovoltaic devices Nano Lett. 10 1302-7

[29] Pisula W, Zorn M, Chang J Y, Mullen K and Zentel R 2009 Liquid crystalline ordering and charge transport in semiconducting materials Macromol. Rapid Commun. 30 1179-202

[30] Hesse H C, Weickert J, Al-Hussein M, Dössel L, Feng X, Müllen K and Schmidt-Mende L 2010 Discotic materials for organic solar cells: effects of chemical structure on assembly and performance Sol. Energy Mater. Sol. Cells 94 560-7

[31] Feng X, Marcon V, Pisula W, Hansen M R, Kirkpatrick J, Grozema F, Andrienko D, Kremer K and Mullen K 2009 Towards high charge-carrier mobilities by rational design of the shape and periphery of discotics Nat. Mater. 8 421-6

[32] Steinhart M, Zimmermann S, Goring P, Schaper A K, Gosele U, Weder C and Wendorff J H 2005 Liquid crystalline nanowires in porous alumina: geometric confinement versus influence of pore walls (vol 5, pg 432, 2005) Nano Lett. 5995

[33] Feng X, Pisula W, Kudernac T, Wu D, Zhi L, De Feyter S and Mullen K 2009 Controlled self-assembly of C3-symmetric hexa-peri-hexabenzocoronenes with alternating hydrophilic and hydrophobic substituents in solution, in the bulk, and on a surface J. Am. Chem. Soc. 131 4439-48 